\title{
Interpolation of erasure bursts via cosine-modulated filterbanks
}

\author{
S JAYASIMHA and C G HIREMATH \\ Signion Systems Pvt. Ltd., 6-3-569/1/2, Rockdale Compound, Somajiguda, \\ Hyderabad 500 082, India \\ e-mail: signion@hotmail.com
}

\begin{abstract}
A novel and low-complexity approach for reconstructing periodic erasure bursts in data sampled at greater than the Nyquist rate, using cosine modulated filterbanks, is described. In the case of interpolation of erasure singlets or doublets periodically repeated over $2 M$ samples, the cosine modulated filterbank approach is shown to have a lower complexity (for a given restoration error) than a standard FIR interpolator. In the case of erasure triplets or quadruplets, periodically repeated over $2 M$ samples, the restoration error is primarily related to whether the $M$-channel filterbank's stopband suppression is better than the condition number of a $2 \times 2$ matrix, where $M$ is determined by the oversampling factor of the data. While the method used for erasure triplets and quadruplets extends to arbitrary erasure bursts, the condition numbers of the associated (larger dimension) matrices deteriorate rapidly with the increase in erasure length, posing practical problems such as the design of very high-attenuation filterbanks and large required implementation wordlengths.
\end{abstract}

Keywords. Chebyshev filters; discrete cosine transforms; equiripple filters; $M$-channel filterbanks; quadrature mirror filters; signal restoration; signal sampling/reconstruction.

\section{Introduction}

The restoration of erasure bursts in oversampled multimedia data has assumed increased significance in the context of cell loss in ATM networks. Many heuristic methods, such as linear interpolation, repetition, or muting, are recommended in many multimedia standards. Other systematic approaches, however, have been considered in the recent years. While Vaidyanathan \& Liu (1988) examine the feasibility of correcting long error bursts in the context of the sampling theorems, Marks and Radbel (Marks 1983; Marks \& Radbel 1984), by considering the condition number of sub-matrices of a DFT matrix, exposed the 
limitation $^{1}$ of the DFT method of signal restoration. The cosine modulated filterbank approach to burst erasure restoration described herein shows similar limitations. However, a poor condition number of a submatrix of a DCT matrix may be overcome by the design of a high stopband attenuation filterbank (in terms of achieving the desired restoration error). It is also shown that the approach proposed applies even to very slightly oversampled signals by increasing the number of channels $M$, albeit at the expense of the condition number of a submatrix of a DCT matrix. The method described is limited by practical considerations such as the design of very high stopband attenuation filterbanks and large required arithmetic word-lengths (for longer erasure bursts in signals approaching the Nyquist bandwidth). The method may be used to correct erasures either for isolated bursts (separated by at least the order of the prototype filter) or for either $M$ - or $2 M$-periodic erasure bursts. The method described is also computationally efficient, and in the simplest single $2 M$-periodic erasure case, obtains a factor of 2 in reduced complexity as compared to an ordinary FIR interpolator.

\section{New restoration method}

The restoration method uses a typical $M$-channel maximally decimated filter bank as shown in figure 1 , where $H_{k}(z)$ and $F_{k}(z), 0 \leq k \leq M-1$ are analysis and synthesis filters, respectively.

Here the cosine modulated filter bank is used which is attractive with respect to implementation cost and design ease. The cosine modulation may be either of type II or type $\mathrm{IV}^{2}$. The impulse response of the analysis and synthesis filters $h_{k}(n)$ and $f_{k}(n)$ are cosine modulated versions of the prototype filter $h(n)$. For type IV cosine modulation:

$$
\begin{aligned}
& \left\{\begin{array}{l}
h_{k}(n)=2 h(n) \cos \left((2 k+1) \frac{\pi}{2 M}\left(n-\frac{N-1}{2}\right)-(2 k+1) \frac{\pi}{4}\right) \\
f_{k}(n)=2 h(n) \cos \left((2 k+1) \frac{\pi}{2 M}\left(n-\frac{N-1}{2}\right)+(2 k+1) \frac{\pi}{4}\right)
\end{array}\right. \\
& \left\{\begin{array}{l}
0 \leq n \leq N-1 \\
0 \leq k \leq M-1
\end{array}\right.
\end{aligned}
$$

where $N$ is the length of $h(n)$. Here the cosine modulation uses a phase shift of $(2 k+1) \pi / 4$ and satisfies the alias cancellation constraint given (Vaidyanathan 1993). The analysis

\footnotetext{
${ }^{1}$ Quoting from Strang \& Nguyen (1996): "The Discrete Cosine Transform (DCT) improves on the DFT for the Same reason that symmetric extension improves upon periodic extension. The symmetric extension is continuous." ${ }^{2}$ The kernel for the modulation matrix is$$
\cos \left[\left((2 k+1) \frac{\pi}{2 M}\left(n-\frac{N^{\prime}}{2}\right)\right) \theta_{k}\right],
$$

where $\theta_{k}$ is the phase shift determined by the alias canceling constraint (Vaidyanathan 1993). For type II modulation, $N^{\prime}$ is the length of the filter and for the type IV modulation, $N^{\prime}$ is the order of the filter.
} 


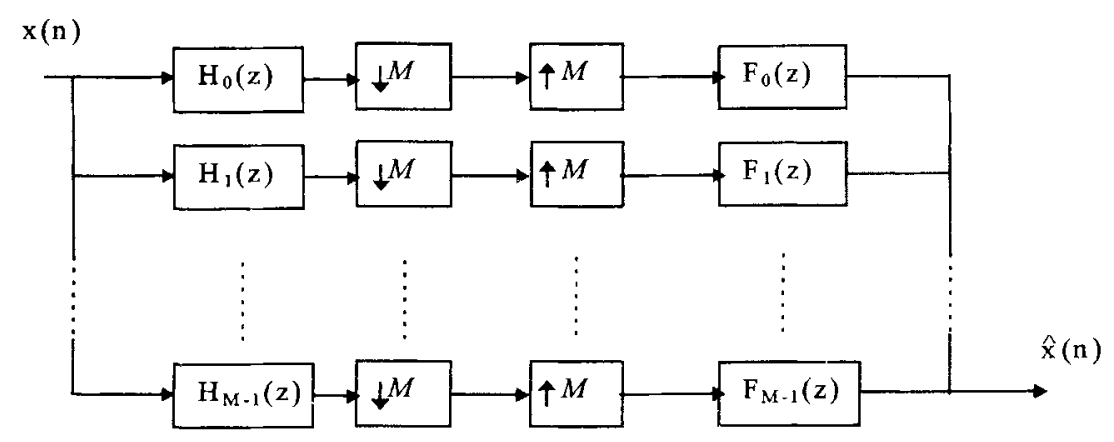

Figure 1. $M$ channel maximally decimated filter bank.

filter with these impulse responses can be separated into $2 M$ polyphase components as

$$
\begin{aligned}
& \begin{aligned}
H_{k}(z)= & \sum_{i=0}^{2 M-1} \sum_{q=0}^{(N / 2 M)-1} 2 h(2 q M+i) \\
& \times \cos \left((2 k+1)\left(i-\frac{N-1}{2}\right) \frac{\pi}{2 M}-(2 k+1) \frac{\pi}{4}\right)(-z)^{-2 q M} z^{-i} \\
= & \sum_{i=0}^{2 M-1} G_{i}\left(-Z^{2 M}\right) \\
& \times \cos \left((2 k+1)\left(i-\frac{N-1}{2}\right) \frac{\pi}{2 M}-(2 k+1) \frac{\pi}{4}\right) z^{-i}
\end{aligned} \\
& \text { where } G_{i}\left(-z^{2 M}\right)=\sum_{\substack{q / 2 M)-1\\
}}^{2 h(2 q M+i)(-z)^{-2 q M}} \\
& \qquad \begin{array}{l}
0 \leq q \leq(N / 2 M)-1 \\
0 \leq k \leq M-1 .
\end{array}
\end{aligned}
$$

For an efficient implementation of this filter bank, the length of the prototype filter $N$ is assumed to be an even multiple of $M$, i.e., $N=2 m M$, where $m$ is an even integer; this condition is not restrictive as a prototype of any length can be padded with an appropriate number of zeroes. This simplifies the filter transfer function to:

$$
\begin{gathered}
H_{k}(z)=\sum_{i=0}^{2 M-1} 2 G_{i}\left(-z^{2 M}\right) \cos \left((2 k+1)(2 i+1-M) \frac{\pi}{4 M}\right) z^{-i} \\
\quad 0 \leq k \leq M-1
\end{gathered}
$$

A fast algorithm for implementing this filter bank using the IDCT-IV like modulation matrix (the modulation matrix used here are same as the DCT-IV matrix without the usual scaling factor for the first coefficient) can be obtained by reordering the polyphase components as follows:

$$
G_{i}^{\prime}= \begin{cases}z^{-M / 2} G_{i+(M / 2)}, & \text { for } i=0.1, \ldots(3 M / 2)-1 \\ -z^{3 M / 2} G_{i-(3 M / 2)}, & \text { for } i=3 M / 2, \ldots, 2 M-1\end{cases}
$$


In terms of this new sequence, the analysis filters are expressed as follows:

$$
\begin{aligned}
H_{k}(z)= & \sum_{i=0}^{M-1} 2\left(z^{-i} G_{i}^{\prime}\left(-z^{2 M}\right)-z^{-(2 M-1-i)} G_{2 M-1-i}^{\prime}\left(-z^{2 M}\right)\right) \\
& \times \cos \left((2 k+1)(2 i+1) \frac{\pi}{4 M}\right), \quad 0 \leq k \leq M-1 .
\end{aligned}
$$

In matrix notation, the analysis filter bank vector $\mathbf{h}(z)$ becomes:

$$
\mathbf{h}(z)=\operatorname{Tg}(z)
$$

where

$$
\begin{aligned}
\mathbf{h}(z) & =\left[\begin{array}{c}
H_{0}(z) \\
H_{1}(z) \\
\cdots \\
H_{M-1}(z)
\end{array}\right], \\
\mathbf{g}(z) & =\left[\begin{array}{c}
G_{0}^{\prime}\left(-z^{2 M}\right)-z^{-(2 M-1) G_{2 M-1}^{\prime}\left(-z^{2 M}\right)} \\
z^{-1} G_{1}^{\prime}\left(-z^{2 M}\right)-z^{-(2 M-2)} G_{2 M-2}^{\prime}\left(-z^{2 M}\right) \\
\ldots \\
z^{-(M-1)} G_{M-1}^{\prime}\left(-z^{2 M}\right)-z^{-M} G_{M}^{\prime}\left(-z^{2 M}\right)
\end{array}\right]
\end{aligned}
$$

and $\mathbf{T}$ is an $M \times M$ IDCT IV like modulation matrix with elements:

$$
t_{k i}=2 \cos \left((2 k+1)(2 i+1) \frac{\pi}{4 M}\right) .
$$

The corresponding synthesis filters $F_{k}(z)$, can also be expressed in terms of the DCT IV like modulation matrix:

$$
\begin{aligned}
F_{k}(z)= & \sum_{i=0}^{M-1} 2 z^{-K}\left(z^{-i} G_{i}^{\prime}\left(-z^{-2 M}\right)-z^{-(2 M-1-i)} G_{2 M-1-i}^{\prime}\left(-z^{-2 M}\right)\right) \\
& \times \cos \left((2 k+1)(2 i+1) \frac{\pi}{4 M}\right), 0 \leq k \leq M-1, \text { where } K=N-2 M
\end{aligned}
$$

In matrix notation, the synthesis filter vector $\mathbf{f}(z)$ is written as

$$
\mathbf{f}^{T}(z)=z^{-N} \mathbf{g}^{T}\left(z^{-1}\right) \mathbf{T}^{T}
$$

where, $\mathbf{f}^{T}(z)=\left[F_{0}(z) F_{1}(z) \cdots F_{M-1}(z)\right]$.

Similarly, the analysis filter and synthesis filter vectors for type II modulation kernel can be expressed in terms of the DCT II modulation matrix as given below:

$$
\mathbf{h}_{I I}(z)=\mathbf{T}_{I I} \mathbf{g}_{I I}(z)
$$

where

$$
\mathbf{h}_{I I}(z)=\left[\begin{array}{c}
H_{0}(z) \\
H_{1}(z) \\
\cdots \\
H_{M-1(z)}
\end{array}\right]
$$




$$
\mathbf{g}_{I I}(z)=\left[\begin{array}{c}
G_{0}^{\prime}\left(-z^{2 M}\right) \\
z^{-1} G_{1}^{\prime}\left(-z^{2 M}\right)-z^{-(2 M-2)} G_{2 M-2}^{\prime}\left(-z^{2 M}\right) \\
\cdots \\
z^{-(M-1)} G_{M-1}^{\prime}\left(-z^{2 M}\right)-z^{-M} G_{M+1}^{\prime}\left(-z^{2 M}\right)
\end{array}\right]
$$

and $\mathbf{T}_{11}$ is an $M \times M$ IDCT II like modulation matrix with elements

$$
t_{\mathrm{ki}}=2 \cos \left((2 k+1) i \frac{\pi}{2 M}\right) \text {. }
$$

The synthesis filter vector $\mathbf{f}(z)$ is written as

$$
\mathbf{f}_{I I}^{T}(z)=z^{-N} \mathbf{g}_{I I}^{T}\left(z^{-1}\right) \mathbf{T}_{I I}^{T}
$$

A polyphase structure, similar to the one given, that implements the analysis filterbank for type IV modulation is shown in figure 2.

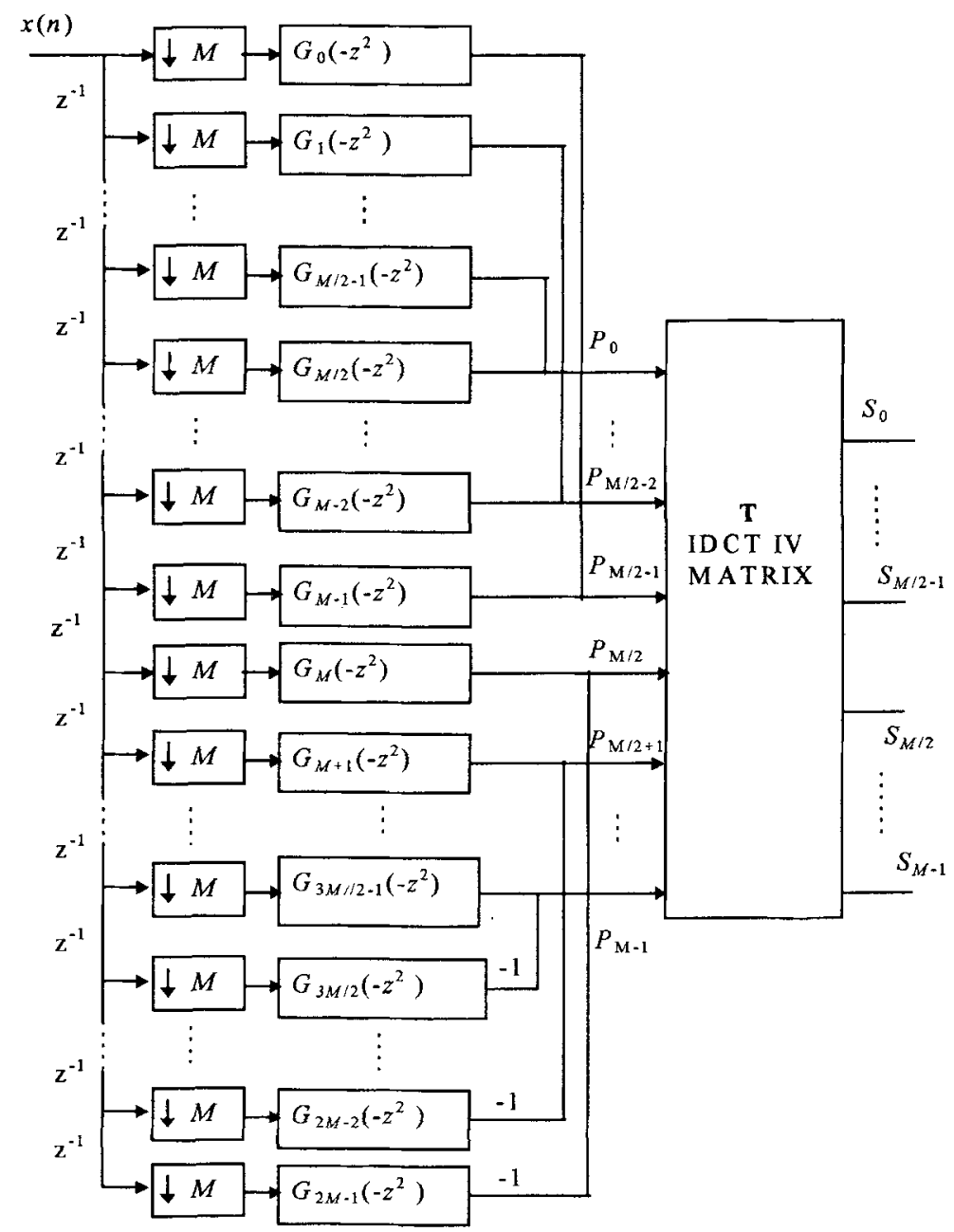

Figure 2. Cosine modulated analysis filter bank in terms of IDCT IV. 
The analysis filters $H_{\mathrm{k}}(z)$ channelize the input signal into $M$ subband signals $S_{\mathrm{k}}$, which are in turn decimated by $M$. These subband components can also be expressed in matrix form in terms of the IDCT matrix and polyphase components as:

$$
\mathbf{S}=\mathbf{T P}
$$

where $\mathbf{S}=\left[S_{0}, S_{1}, \ldots, S_{M-1}\right]^{T}$ is a vector of subband components $S_{k}$ and $\mathbf{P}=\left[P_{0}, P_{1}\right.$, $\left.\ldots, P_{M-1}\right]^{T}$ is the vector of polyphase components $P_{\mathrm{i}}$ (i.e., $\mathbf{P}=\mathbf{g}(z) \mathbf{X}(z)$, where $\mathbf{X}(z)=$ $\left.\left[x(z) z^{-1} x(z) \cdots z^{-M-1} x(z)\right]^{T}\right)$ and $\mathbf{T}$ is a $M \times M$ orthogonal IDCT IV matrix. For an oversampled signal with a normalized bandwidth of $\mathrm{r}\left(2 . F / F_{\mathrm{S}}\right.$, where $F$ is the bandwidth of the signal and $F_{\mathrm{S}}$ is the sampling frequency), and the last $K=[(1-r) \cdot M-1\rceil$ subband samples are not present.

The $p$ missing (or lost) samples are so aligned that a minimum number of polyphase components are unknown. In the case of a burst of erasures, the erased sample indices range from $M-p / 2(\bmod M)$ to $M+p / 2(\bmod M)$, so that only $\lceil p / 2\rceil$ polyphase components are unknown. Then the number of channels of the filter bank $M$ are chosen according to:

$$
M=\left\lceil\frac{(p+1)}{2} /(1-r)\right]_{2},
$$

where, \lceil\rceil$_{2}$ denotes rounding to an even number towards infinity.

For the filterbank with DCT-II modulation matrix, the number of unknown polyphase components is $\lceil(p+1) / 2\rceil$. Therefore, when the length of erasure burst is even the number of unknown polyphase components for the filter bank with DCT-IV modulation matrix is one less than that for the filter bank with DCT-II modulation matrix.

Next, split the polyphase vector into a vector of known components $\mathbf{P}_{1}$ and a vector of unknown components $\mathbf{P}_{2}$, so that

$$
\left[\begin{array}{l}
\mathbf{S}_{1} \\
\mathbf{S}_{2}
\end{array}\right]=\left[\begin{array}{ll}
\mathbf{T}_{11}^{p} & \mathbf{T}_{12}^{p} \\
\mathbf{T}_{21}^{p} & \mathbf{T}_{22}^{p}
\end{array}\right]\left[\begin{array}{l}
\mathbf{P}_{1} \\
\mathbf{P}_{2}
\end{array}\right]
$$

where, $\mathbf{T}_{11}^{p}, \mathbf{T}_{12}^{p}, \mathbf{T}_{21}^{p}$ and $\mathbf{T}_{22}^{p}$ are the submatrices of a permuted IDCT IV matrix. The columns of the modulation matrix $\mathbf{T}$ are permuted according to the known and unknown polyphase components. As the input signal is bandlimited, the subband component vector $\mathbf{S}_{2}$ can be set to zero. This results in a system of linear equations which are solved to determine the unknown polyphase components $\mathbf{P}_{2}$.

$$
\left[\mathbf{T}_{22}^{p}\right] \mathbf{P}_{2}=-\left[\mathbf{T}_{21}^{p}\right] \mathbf{P}_{1}
$$

As shown in figure 2, when the erased sample burst indices range from $M-p / 2(\bmod M)$ to $M+p / 2(\bmod M)$, the first $p / 2$ components of the polyphase vector $\mathbf{P}_{2 F}=\left[P_{0}, P_{1}, \ldots\right.$, $\left.P_{p-1}\right]$ will have to be determined for the first $M$-sample input block. When the next $M$-sample block is the input, the last $p / 2$ components of the polyphase vector $\mathbf{P}_{2 L}=$ $\left[P_{M-p-1}, P_{M-p}, \ldots, P_{M-1}\right]$ are unknown. The unknown polyphase component vectors alternate in this manner until all the erased samples are restored.

In the preceding paragraphs, we have considered $2 M$ periodicity in determining $M$ from the given normalized bandwidth of $r$ and burst length $p$. An alternate view of figure 2 is to consider a periodicity of $M$ in determining $M$ from the oversampling factor and burst 
length. In this case, each missing poly phase component in the top $M$ polyphase components $G_{j}\left(-z^{2 M}\right)$, has a counterpart, separated by $M$ from it, that is also missing. In this case, the DCT submatrix is always constant for every $M$-sample block during the restoration process. The number of subbands $M$ is determined by

$$
M=\left\lceil\frac{p+1}{1+r}\right\rceil_{2} \text {. }
$$

The number of channels of the analysis filter bank obtained using (15) is less than twice the number of channels obtained using (12). Therefore, the interval between the error bursts using $2 M$-periodicity (with $M$ given by (12)) can, in some cases, be reduced by using $M$-periodicity (with $M$ given by (15)).

\section{Error in restoration of erased samples}

The estimated polyphase components are passed through the polyphase structure of the synthesis filter bank to determine the erased samples. Therefore, the error in the restored samples is given by:

$$
\|\delta \mathbf{x}\|=\| \mathbf{g}_{e}\left(z^{-1} \delta \mathbf{P}_{2}(z)\|\leq\| \mathbf{g}_{e}\left(z^{-1}\right)\|\| \delta \mathbf{P}_{2}(z) \|\right.
$$

where

$$
\left\|\delta P_{2}(z)\right\|=\left\|\begin{array}{l}
\delta \mathbf{P}_{2 F}+z^{-2} \delta \mathbf{P}_{2 F}+\cdots z^{-\frac{N}{2 M}} \delta \mathbf{P}_{2 F} \\
\delta \mathbf{P}_{2 L}+z^{-2} \delta \mathbf{P}_{2 L}+\cdots z^{-\frac{N}{2 M}} \delta \mathbf{P}_{2 L}
\end{array}\right\|
$$

and $\left\|\mathbf{g}_{e}\left(z^{-1}\right)\right\|$ is the norm of the polyphase components in $g(z)$ corresponding to $\mathbf{P}_{2 F}$ and $\mathbf{P}_{2 L}$.

The error of the estimates of the unknown polyphase components using (14) is due to the error introduced into (14) by the assumption that the subband vector $\mathbf{S}_{2}$ is zero. The error in the restoration of $\mathbf{P}_{2}, \delta \mathbf{P}_{2}$ satisfies:

$$
\left\|\delta \mathbf{P}_{2}\right\| \leq \kappa\left(\mathbf{T}_{22}^{p}\right) \frac{\left\|\mathbf{S}_{2}\right\|}{\left\|\mathbf{T}_{21}^{p} \mathbf{P}_{1}\right\|}\left\|\mathbf{P}_{1}\right\|
$$

where, $\kappa\left(\mathbf{T}_{22}^{p}\right)$ is the condition number of matrix $\mathbf{T}_{22}^{p}{ }^{3}$ and \|\|$^{4}$ denotes the norm of matrix or a vector (Kreyzig 1993). For error vector $\delta \mathbf{T}_{22}^{p}$ to be small, both the condition number of $\mathbf{T}_{22}^{p}$ and the norm subband vector $\mathbf{S}_{2}$ should be small. If the condition number of the matrix $\mathbf{T}_{22}^{p}$ can be controlled (through the choice of $M$ ), the norm of the subband vector $\mathbf{S}_{2}$ can be reduced by using a filter bank with higher stop-band attenuation. ${ }^{5}$ It should also be noted that as the condition number of the matrix $\mathbf{T}_{22}^{p}$ increases. the word-length required

\footnotetext{
${ }^{3}$ The condition number of matrix $\mathbf{A} \kappa(\mathbf{A})$, is the ratio of largest singular value of $\mathbf{A}$ is the largest singular value of $\mathbf{A}$.

${ }^{4}$ Norm of matrix $\mathbf{A}$ is the largest singular value of $\mathbf{A}$.

${ }^{5}$ Assuming a perfectly bandlimited signal in $(0 .(M-K) \pi)$, the $L^{\infty}$ norm of the decimated signal in any high subband (that will he set to zero) has an upper bound of $M-K$ times the supremum of the $L^{\infty}$ norms of the signals in the first $M-K$ subbands multiplied by the supremum of stopband gain of the $K$ high subband filters. This again will be small if the maximum stopband gain of the prototype is small.
} 
Table 1(a). Condition numbers for DCT II and DCT IV submatrices $\mathbf{T}_{22}^{p}$ for different $M$ with $K=2$.

\begin{tabular}{rcccc}
\hline$M$ & $\kappa\left(\mathbf{T}_{22}^{p}\right)$ & $\kappa\left(\mathbf{T}_{22}^{p}\right)$ & $\kappa \mathbf{T}_{22}^{p}$ & $\kappa\left(\mathbf{T}_{22}^{p}\right)$ \\
& $\mathbf{P}_{2 F}$ & $\mathbf{P}_{2 L}$ & $\mathbf{P}_{2 F}$ DCT & $\mathbf{P}_{2 L}$ \\
& DCT IV & DCT IV & II & DCT II \\
\hline 8 & 91.2789 & 11.2485 & 24.4259 & 41.5830 \\
12 & 225.6398 & 27.2672 & 56.7647 & 108.2118 \\
24 & 954.6982 & 114.6825 & 231.7052 & 472.3874 \\
32 & 1711.200 & 205.4472 & 413.3550 & 850.5666 \\
\hline
\end{tabular}

Table 1(b). Condition numbers for DCT II and DCT IV submatrices $\mathbf{T}_{22}^{p}$ for different $K$ with $M=8$. $\mathbf{T}_{22}^{p}$ for different $M$ with $K=2$.

\begin{tabular}{lcccc}
\hline$K$ & $\kappa\left(\mathbf{T}_{22}^{p}\right)$ & $\kappa\left(\mathbf{T}_{22}^{p}\right)$ & $\kappa \mathbf{T}_{22}^{p}$ & $\kappa\left(\mathbf{T}_{22}^{p}\right)$ \\
& $\mathbf{P}_{2 F}$ & $\mathbf{P}_{2 L}$ & $\mathbf{P}_{2 F}$ DCT & $\mathbf{P}_{2 L}$ \\
& DCT IV & DCT IV & II & DCT II \\
\hline 2 & 91.2789 & 11.2485 & 24.4259 & 41.5830 \\
3 & 667.0022 & 40.2057 & 133.4108 & 190.1040 \\
4 & 1059.4000 & 58.7428 & 260.1650 & 236.5874 \\
\hline
\end{tabular}

for solving (14) also increases and imposes practical constraints on use of the usefulness of the algorithm. In the case of a large erasure burst, the reconstruction (psuedo-QMF, perfect, or near-perfect) properties of the prototype filter has only a negligible effect on restoration error. ${ }^{6}$ Consequently, in the following examples used to illustrate lost-sample restoration, we have used the near-equiripple pseudo-QMF bank designed using the method described in by Jayasimha \& Hiremath (1998).

The condition number of the matrix $\mathbf{T}_{22}^{p}$ deteriorates with increasing $M$ and with the number of missing polyphase components (i.e., the length of the lost-sample burst). The condition number of the matrix $\mathbf{T}_{22}^{p}$ used while estimating $\mathbf{P}_{2 F}$ and $\mathbf{P}_{2 L}$ vectors is tabulated in tables $1 \mathrm{a}$ and $\mathrm{b}$, respectively. As can be observed from the table, the maximum condition number is for the DCT-IV modulation matrix. Therefore, when the condition number is large, the filter bank with the DCT-II modulation matrix is used (in particular, for odd $p$ ).

To compensate for the ill-conditioning of matrix $\mathbf{T}_{22}^{p},\left\|\mathbf{S}_{2}\right\|$ should be small and the prototype filter must be designed to have high stopband attenuation. A method to design high-order, large even $M$, filterbanks (where $M$ is an even composite number) is discussed by Hiremath \& Jayasimha (1997).

\section{Interpolation of periodic erasure singlets and doublets}

A block diagram for the efficient restoration of periodic, erasure singlets, compared with the restoration of periodic erasure singlets using a type II maximally-decimated $M$-channel (where $M$ is divisible by 4 ) cosine modulated filterbank, is illustrated by an example in figure 3 for periodic erasure singlets with periodicity of $I=4$ and $M=12$.

\footnotetext{
${ }^{6}$ In pseudo-QMF banks, the stopband attenuation; however, the restoration error is of the order of the stopband attenuation
} 
Table 2. Restoration ESR for erasure bursts of various lengths $(M=8)$.

\begin{tabular}{lcccc}
\hline $\begin{array}{l}\text { Erasure } \\
\text { burst }\end{array}$ & $\begin{array}{c}\text { Actual } \\
\text { samples }\end{array}$ & $\begin{array}{c}\text { Restored } \\
\text { samples }\end{array}$ & $\begin{array}{c}\text { ESR upper } \\
\text { bound }\end{array}$ & $\begin{array}{c}\text { ESR } \\
\text { practical }\end{array}$ \\
\hline $\begin{array}{l}\text { Singlet } \\
\text { Doublet }\end{array}$ & 3352 & 3353 & 0.0026 & 0.00029 \\
Triplet & 3666,3352 & 3668,3345 & 0.004629 & 0.001465 \\
Quadruplet & $38666,3352,2855$ & $3668,3345,2873$ & 0.006688 & 0.001317 \\
Quintuplet & $3806,3666,335352,28555$ & $3779,3651,3352,2871$ & 0.005827 & 0.005057 \\
\hline
\end{tabular}

The complexity per interpolated point for the restoration of singlets with $M=12, I=4$ and $W=128$ is given by $C=I / M\left(\mathrm{DCT}_{\mathrm{IV}}^{6}+\mathrm{DCT}_{\mathrm{IV}}^{3}+3 \cdot\right.$ adds $\left.+\mathrm{DCT}_{\mathrm{II}}^{3}\right)+W / M$ multiply adds $\approx 25$ multiplications +35 additions.

The usual FIR interpolator derived from a 4th band filter (Adams 1991) for the restoration (with similar performance) of these periodic erasure singlets has 89 taps and uses 67 additions and 34 multiplications.

In a similar manner, doublets can be restored using the modulation matrix of DCT IV for which no FIR interpolator is proposed by Adams (1991). Note that the condition number $\kappa\left(\mathbf{P}_{22}^{p}\right)$ is one and the error in the estimation depends upon the subband component $S_{\mathrm{M}-1}$.

\section{Erasure burst restoration example}

Some randomly selected position in audio data sampled at $44.1 \mathrm{kHz}$ (an example from "Dire Straits'), bandlimited to $13.23 \mathrm{kHz}$, is used to test the described restoration method. A $M=8, N=128$ low-pass prototype with specifications: Passband edge normalized frequency: 0.01042 Stopband edge normalized frequency: 0.11458 Stopband attenuation: $-80 \mathrm{~dB}$ was designed (Jayasimha \& Hiremath 1998). This odd-length prototype padded with a leading zero, and used with a DCT-II modulation matrix for fast implementation, has a reconstruction error of $-54 \mathrm{~dB}$. The average restoration error to signal ratio, a measure

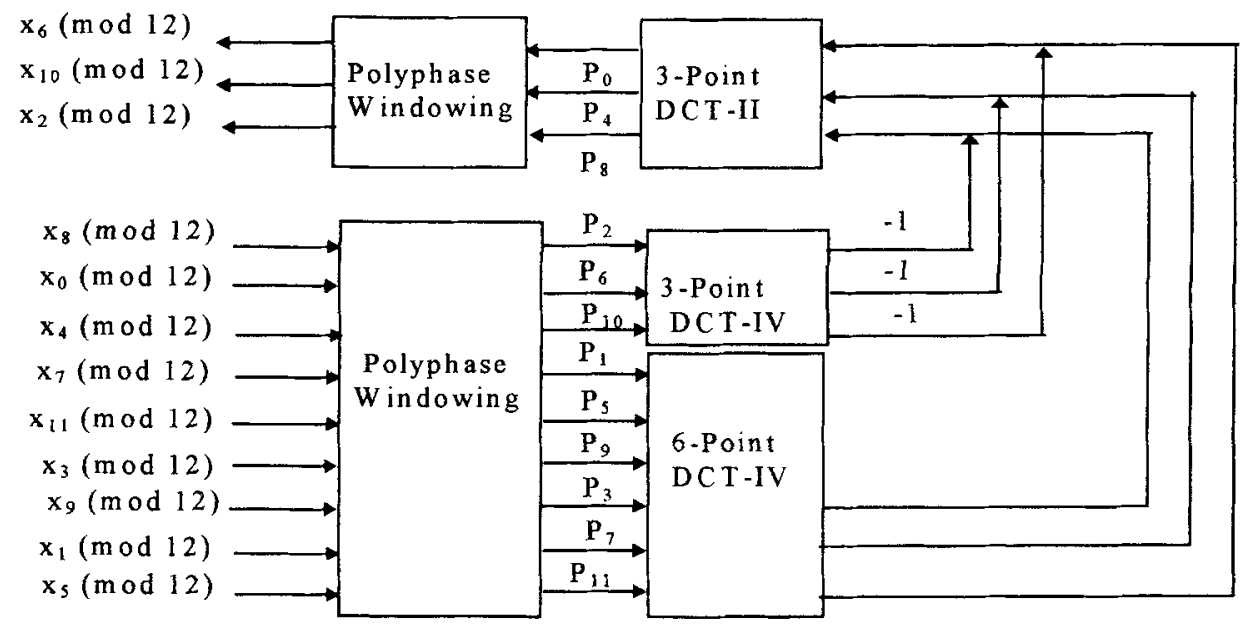

Figure 3. Restoration of singlet $s(M=12, I=4)$. 
of the restoration's accuracy, is:

$$
E S R=\left(\left\{\sum_{n}(x(n)-\hat{x}(n))^{2}\right\} /\left\{\sum_{n} x^{2}(n)\right\}\right)^{1 / 2} .
$$

The restoration ESR's for isolated bursts of various lengths for $M=8$ are given in table 2 .

\section{Conclusion}

A novel, low-complexity cosine-modulated filterbank approach to short-burst erasure interpolation has been described. Potential applications areas of short-erasure burst restoration are in removal of lightning-induced impulsive noise in VLF and LF communications (detected by short-duration saturation of amplifiers and ADCs) and the extension of the ADC dynamic range when the bandlimited signal being acquired is slightly oversampled. One example of the latter application is that saturated signals in short regions of some 16-bit compact disk recordings may be restored and played back on 18-bit DACs.

\section{References}

Adams R W 1991 Non-uniform sampling of audio signals. Preprint 3140 (E-1) of the 91 st convention of the Audio Engineering Society, New York

Hiremath C G, Jayasimha S 1997 Design of large order prototype filter for composite M-channel filterbanks. Proc. of the Third National Conference on Communications and Networking, NCC97, pp 63-65

Jayasimha S, Hiremath C G 1998 Pseudo-QMF banks with near-equiripple performance. IEEE Trans. Signal Process. SP-46: 209-214

Kreyszig E 1993 Advanced engineering mathematics (New York: John Wiley \& Sons) p 998

Marks R J II Restoring lost samples from an oversampled band-limited signal. IEEE Trans. Acoust. Speech Signal Process. ASSP-31: 752-755

Marks R J II, Radbel M 1984 Error of linear estimation of lost samples in an oversampled bandlimited signal. IEEE Trans. Acoust. Speech Signal Process. ASSP-32: 648-654

Strang G, Nguyen T 1996 Wavelets and filter banks (Wellesley, MA: Wellesley-Cambridge Press) pp 276-287

Vaidyanathan P P 1993 Multirate systems and filter banks Signal Processing Series (Englewood Cliffs, NJ: Prentice Hall) pp 370-372

Vaidyanathan P P, Liu V C 1988 Classical sampling theorems in the context of multirate and polyphase digital filter structures. IEEE Trans. Acoust. Speech Signal Process. ASSP-36: $1480-1495$ 